\title{
Evaluation of Several Dosimeters for Identification of Irradiated Foods Using a 5 MeV Electron Beam
}

\author{
Makoto Miyahara, ${ }^{*}, a$ Hitoshi Ito, ${ }^{b}$ Kouji Ueno, ${ }^{c}$ Yutaka Yamase, ${ }^{c}$ and Masatake Toyoda ${ }^{a}$ \\ ${ }^{a}$ National Institute of Health Sciences, 1-18-1 Kamiyoga, Setagaya-ku, Tokyo 158-8501, Japan, Japan Atomic Energy Research In- \\ stitute Takasaki Establishment, 1233 Watanuki-mati, Takasaki City, Gunma 370-1292, Japan, and 'Japan Irradiation Service Co., \\ Tsukuba Electron Irradiation Center, 4-16 Midorigahara Tsukuba City, Ibaraki 300-2646, Japan
}

(Received September 5, 2001; Accepted October 13, 2001)

\begin{abstract}
Electron-beam irradiation facilities are commercially utilized for food irradiation. This study investigates whether dosimeters used to detect gamma-rays can also be used to accurately detect electron-beam irradiation. The irradiation souses for gamma-ray at the Takasaki Institute were used throughout this study. Four kinds of dosimeters, RadiaChromic (RC), GammaChromic (GC), Amber3042 (AM), Radix (RX), and alanine (AL) were irradiated at doses from 0.1 to $60 \mathrm{kGy}$ using a $5 \mathrm{MeV}$ electron-beam (EB) at the Japan Irradiation Service Co. Ltd. (Japan). AL was submitted to the National Physics Laboratory in order to evaluate the doses that AL were absorbed. At low-level dose range, all dosimeters indicated correct values. This means those dosimeters were usable to accurately detect electron beam irradiation. But at higher range they all required correction due to either temperature effects or varying sensitivity to electron-beam exposure. Dose-depth profiles were obtained using ham and cheese samples, in order to check penetration of EB by the calibrated machine.
\end{abstract}

Key words — electron-beam irradiation, dosimeter, food irradiation, calibration

\section{INTRODUCTION}

Electron-beam irradiation of food has been commercially utilized in the United States since 2000. The advantages of irradiating with electron beam (EB) against gamma-sources have been well-established. ${ }^{1)}$ For example, EB irradiation can be controlled by switch-operation. Even more importantly, the irradiation range can be varied by changing the acceleration voltage, such that EB irradiation can deliver the required energy where it is needed. On the other hand, the EB range can be affected by the target (i.e., irradiated material). The thicker, higher density, and greater atomic number of component elements the target contains, the shorter will be EB range. It is particularly difficult to accurately estimate the EB range in foods which consisting of inhomogeneous materials. ${ }^{2)}$ Moreover, when food is irradiated with high-energy EB of over $10 \mathrm{MeV}$, it is difficult to neglect the effects of X-ray beamstrahlung or of the radioactivities induced which are formed by $(\gamma, n)$ and $(n, \gamma)$ reactions. ${ }^{3,4)}$ Therefore, it is necessary to consider these side effects of EB ir-

\footnotetext{
*To whom correspondence should be addressed: National Institute of Health Sciences, 1-18-1 Kamiyoga, Setagaya-ku, Tokyo 158-8501, Japan. Tel.: +81-3-3700-1141; Fax: +81-3-37076950; E-mail:mmiyaha@nihs.go.jp
}

radiation together with the temperature effects when performing EB dosimetry with chemical dosimeters. The operation parameters, affect "apparent absorbed dose." ${ }^{5)}$

Dose rate of EB is greater than that of gammaray irradiation, and is over $10 \mathrm{MGy}$ giving dose rate effects on dosimeters. The chemical dosimeters are based on absorbance change of dosimeter element, that caused by radio-chemical reaction. Therefore, many parameters related irradiation conditions, such as partial pressure of oxygen, temperature, humidity and so on, can affect "apparent dose" and cause error in measuring absorbed dose.

For many years, food irradiation performed using cobalt-60 source with dosimeter for gamma-ray. Those dosimeter systems are established and used in any conditions which food irradiation required. However, there is no dosimeter to detect EB irradiation. It may be convenient to use such dosimeters, RadiaChromic dosimeter (RC, range, 0.5-200 kGy), Gammachrom YR, (GC, range, 0.1-3 kGy), Amber 3042 (AM, Range, 1-30 kGy), and Radix Dosimeter (RX, Range: 5-50 kGy) for detection of absorbed dose by irradiation with EB.

In the present method, the absorbance change of these practical dosimeters by irradiation is measured, and then the absorbed dose is calculated us- 
ing a calibration curve. The calibration curve is constructed as follows: First, using a standard source such as the National Physics Laboratory (NPL) of U.K., dosimeters, including an AL dosimeter are irradiated at several doses, and the absorbance of the dosimeters are related to the given doses. ${ }^{6}$ Commercially available dosimeters are calibrated by irradiation of cobalt 60 . The energy of the gamma-rays is about $1.13 \mathrm{MeV}$ and the irradiation range is longer than that of EB irradiation because the EB is a higher energy transfer (LET) source. Therefore, in the case that dosimeters are used for gamma-ray irradiation, it is not necessary to consider the energy reduction due to self-absorption. On the other hand, when dosimeters are used for EB measurement, they show limitations due to the nature of EB irradiation. As discussed above, the range of EB is affected greatly by the irradiation conditions and the thickness of the dosimeter. To avoid complication due to thickness, for measurement of EB irradiation tend to be very thin, called as film dosimeters (RC, GAF chromic, CTA, etc.). However, the measurable ranges of these are not suitable for food irradiation monitoring. Their most inconvenient properties are the sensitivity to light, temperature, and humidity. One possible solution for measurement of absorbed doses of food irradiation by EB irradiation is the use of gammaray dosimeters which have a long and established history and are easy to handle. Such dosimeters could be used for measurement of irradiation with EB if it could be confirmed that they yield values comparable to those given by alanine dosimeter (AL) under equivalent irradiation conditions. In developing detection methods for irradiation with EB, it is important to determine accurate absorbed doses in food samples. In this study, several dosimeters were evaluated using $5 \mathrm{MeV}$ EB under same conditions and dose-depth profiles in some foods were measured.

\section{MATERIALS AND METHODS}

Dosimeters — The dosimeters used were RC dosimeter (FWT81, Bach8F8; range, 0.5-200 kGy), FWT, U.S.A.; GC YR (Bach 6; range, 0.1-3 kGy), Hawell, U.K.; AM (Bach M, Range, 1-30 kGy), Hawell, U.K.; RX Dosimeter (Bach 6; Range: 5-50 kGy), Radie, Takasaki; AL, NPL, U.K.

Samples — As food samples ready-to-eat packages of ham and cheese were purchased at a local supermarket. The samples were cut into slices of uniform thickness (ham, $2.3 \mathrm{~mm}$; cheese, $2.7 \mathrm{~mm}$ ).
A vernier caliper gauge was used to measure the thickness. Five ham and cheese slices were measured, and those measurements were averaged.

\section{Apparatus}

Irradiation Equipments: The Cobalt-60, platewet-type irradiator of the Japan Atomic Energy Institute, Takasaki Establishment was used for irradiation. The RDI model, $5 \mathrm{MeV}$ dynamitoron accelerator of the Japan Irradiation Service Inc. Co. (JISCO), Tsukuba Facility was used. Cobalt-60 Table stick dry-type irradiator, of the Tokyo Metropolitan Industry and Technology Institute, Komazawa Facility, was also used.

Spectrophotometer: A Hitachi model GenSpecIII was used at $600,520,603$, and $320 \mathrm{~nm}$ for RC, GC, $\mathrm{AM}$, and RM, respectively.

Thickness Gage: A Mitutoyo model, Kawasaki, Japan, was used.

\section{Irradiation Procedure}

Dosimeter: Treatment by cobalt-60: each dosimeter was irradiated at $6 \mathrm{kGy} / \mathrm{hr}$ at room temperature (about $25^{\circ} \mathrm{C}$ ). Treatment by EB: each dosimeter was irradiated at $10 \mathrm{GGy} / \mathrm{hr}$ on ice bath (about $0^{\circ} \mathrm{C}$ ). Dose was average of three determination with three dosimeter elements.

Food with EB: RC dosimeters were set between food slices. The multi-layer consisted with food slices and dosimeters were placed in an ice bath. The instrumental parameter set was follows: acceleration voltage, $5 \mathrm{MeV}$; current, $1.56-5.44 \mathrm{~mA}$; cart speed, $7.5-15 \mathrm{~m} / \mathrm{sec}$.

\section{RESULTS AND DISCUSSION}

\section{Calibration of EB Equipment}

RC dosimeters and AL dosimeters were irradiated at room temperature in the range of 0 to $60 \mathrm{kGy}$. The AL dosimeters were sent back to NPL in order to be evaluated the absorbed doses. The EB equipment and RC dosimeters were calibrated according to the results. The results are shown in Fig. 1 with the horizontal axis being the nominal dose and the vertical axis being the NPL values. A good linear relationship was observed, indicating that the operation, calibration, and measurement were stable and accurate.

\section{Calibration of Dosimeters by Irradiation of Co- balt-60 \\ Before using a dosimeter, it is critical to cali- brate it using a standard irradiation source, such as}




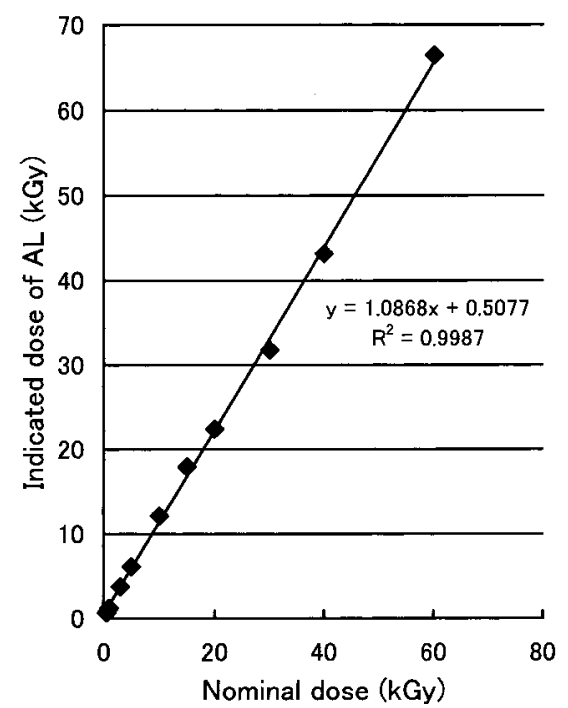

Fig. 1. Corrected Dose of AL Dosimeter by NPL vs. Nominal Dose with Irradiation of $5 \mathrm{MeV} \mathrm{EB}$

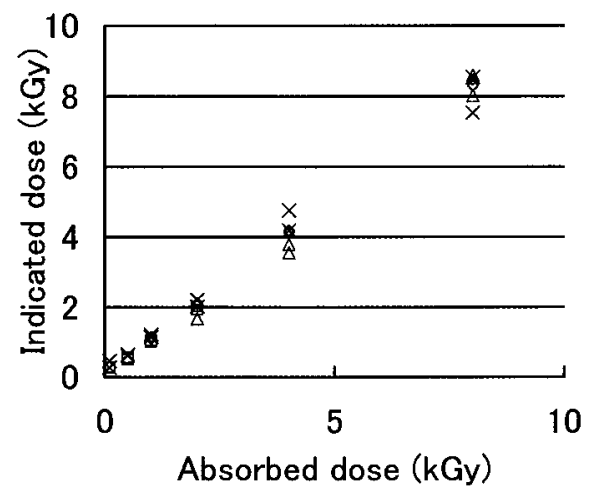

$\circ A M, y=1.0423 x+0.0365 ; R 2=0.9996$

$\square$ GC, $y=0.9135 x+0.1274 ; R 2=0.9939$ $\triangle R X, y=1.1153 x-0.6595, R 2=0.9946$ $X R C_{,} y=0.9910 x+0.2229 ; \quad R 2=0.9896$

Fig. 2. Indicated Doses of Dosimeters with Gamma-Ray Irradiation of ${ }^{60} \mathrm{Co}$

an NPL. However, this method is expensive and fairly slow. For daily calibration, an easy and rapid procedure is desired. An AL dosimeter is some times used for the purpose, but is still cost-prohibitive. ${ }^{7)}$ Daily calibration could perhaps be best accomplished using an gamma-ray source that is periodically calibrated. In this study, using a gamma source equipped in the Japan Atomic Energy Institute, AM, GC, RX, and $\mathrm{RC}$ dosimeters were examined. As shown in Fig. 2, the calculated doses of all dosimeters were linear to the given doses, using the absorbance-dose equations (calibration equation) provide by the manufacturers. The slopes of the dose response curve ranged from 0.91 to 1.11 and $\mathrm{R}^{2}$ ranged from 0.9896

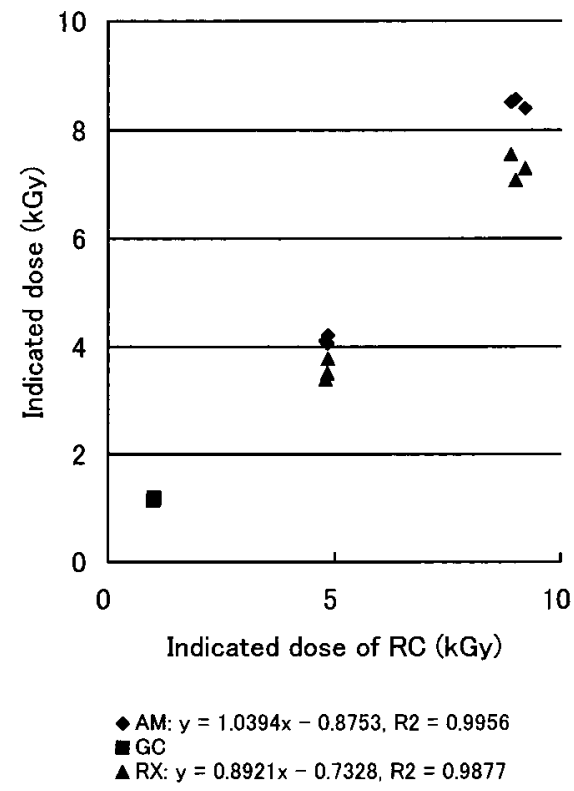

Fig. 3. Indicated Dose of RC vs. AM, GC, and RX with $5 \mathrm{MeV}-$ EB Irradiation

to 0.9996 . Based on these results, the calibration equations are adequate for determination of the dose by gamma-irradiation. These results also show that none of dosimeters used in this study were compromised by long-term storage.

\section{Comparison of AM, GC, and RX Dosimeters with RC Dosimeter, Using EB Irradiation}

The dose responses of the gamma-ray-calibrated dosimeters were examined at room temperature using $5 \mathrm{MeV}$ EB irradiation. Because the stability of EB irradiation is less than that of gamma-ray irradiation, it is necessary to check the absorbed dose at every irradiation. In our study, an RC dosimeter that was traceable to a standard source was used as a reference dosimeter.

The performance of all dosimeters for gammarays (AM, GC, RX) was examined under EB irradiation condition. The results are shown in Fig. 3.

The $\mathrm{R}^{2}$ value and slope of AM were 0.9956 and 1.0394 , respectively and these results shows good correlation to the absorbed doses. This means that even though the thickness of the AM dosimeter is about $3 \mathrm{~mm}$, AM can be used to measure the absorbed dose under irradiation by EB. The linearity of RX was not good, so that this dosimeter required another calibration curve for EB. GC dosimeter also required a calibration curve for $\mathrm{EB}$.

Difference in sensitivity of dosimeters was thus related to the difference of the dose rates between EB and gamma-rays. 


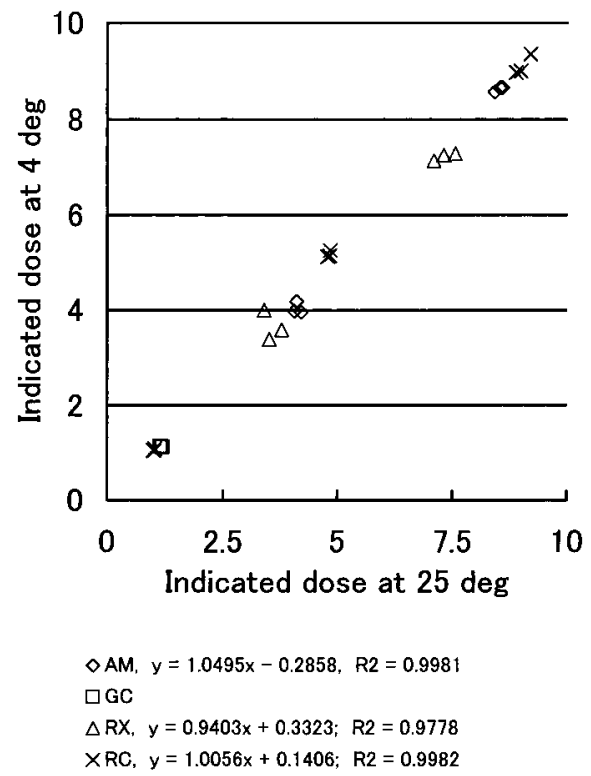

Fig. 4. Temperature Effect on Indicated Doses of AM, GC, RX, and $\mathrm{RC}$ with Irradiation of $5 \mathrm{MeV} \mathrm{EB}$

\section{Temperature Effect}

Food irradiation is performed at a variety of temperatures as required to maintain the quality of individual foods. In this study, AM, GC, RX, and RC dosimeters were irradiated at 4 and $25^{\circ} \mathrm{C}$ to examine their performance.

The calibration curves used were those provided by the manufacturers. RX was given two calibration curves for irradiation at 0 and at $19^{\circ} \mathrm{C}$. The results are shown in Fig. 4, and indicated that there was a relationship between the dose responses at $4^{\circ} \mathrm{C}$ and $25^{\circ} \mathrm{C}$. The horizontal axis represents the indicated dose of dosimeter at $25^{\circ} \mathrm{C}$ and the vertical axis represented that at $4^{\circ} \mathrm{C}$. The slopes of the lines of both $\mathrm{AM}$ and $\mathrm{RC}$ were about $1\left(\mathrm{R}^{2}=0.998\right)$, which means that the indicated doses at $4^{\circ} \mathrm{C}$ and $25^{\circ} \mathrm{C}$ were practically identical. This illustrats that in this temperature range, no correction was needed to cancel the temperature effects. AM and RC are both characterized by a stability of temperature. The slope for the RX dosimeter was 0.9403 , which means that the indicated dose was greater at $25^{\circ} \mathrm{C}$ than that at $4^{\circ} \mathrm{C}$. This result indicates the calibration curves provided were not adequate for our temperature conditions and that RX required more critical temperature correction. In addition, the sensitivity of the RX dosimeter is varied from element to element, possibly due to limitations in quality control. GC was designedch for irradiation at low temperatures, therefore, performed as expected.

From the results, $\mathrm{RC}, \mathrm{GC}$, and $\mathrm{AM}$ can be used

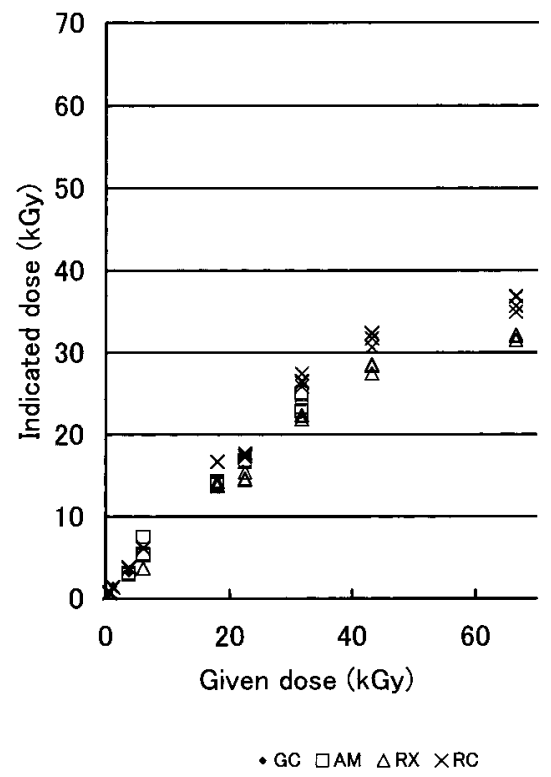

Fig. 5. Dose-Response of Dosimeters at High Dose Levels with Irradiation of $5 \mathrm{MeV} \mathrm{EB}$

to detect low level EB irradiation.

\section{Response of Dosimeters at High-Level EB Irra- diation}

The WHO has recommended that limitations of dose should not be set for food irradiation. In keeping with this recommendation, the dosimeter responses of the dosimeters at both high- and lowdoses were analyzed. An AL and four other dosimeters were irradiated up to $60 \mathrm{kGy}$ at room temperature together. Calibration curves of the GC, RC, RX, and $\mathrm{AM}$ dosimeters were obtained at the range of 0 to $10 \mathrm{kGy}$, at room temperature (about $23^{\circ} \mathrm{C}$ ).

The results are shown in Fig. 5. The horizontal axis corresponds to the corrected dose measured by the AL dosimeter (traceable to gamma source at the NPL), and the vertical axis to the indicated dose of GC, RC, RX, and AM. All dosimeters required collection at above $40 \mathrm{kGyd}$. The measurable range of each dosimeter was dependent on the power of the photometer used, and was restricted by the absorbance of the irradiated dosimeter element. Thus, the deviation of the dose-response curve at high dose range may have been caused by the non-linear response character of the dosimeter and/or the limitations of the photometer.

\section{Dose-Depth Profile of Food}

The dose-distribution of food samples (targets) was determined after irradiation of EB irradiation. The resulting "dose-depth profile" is a function of 


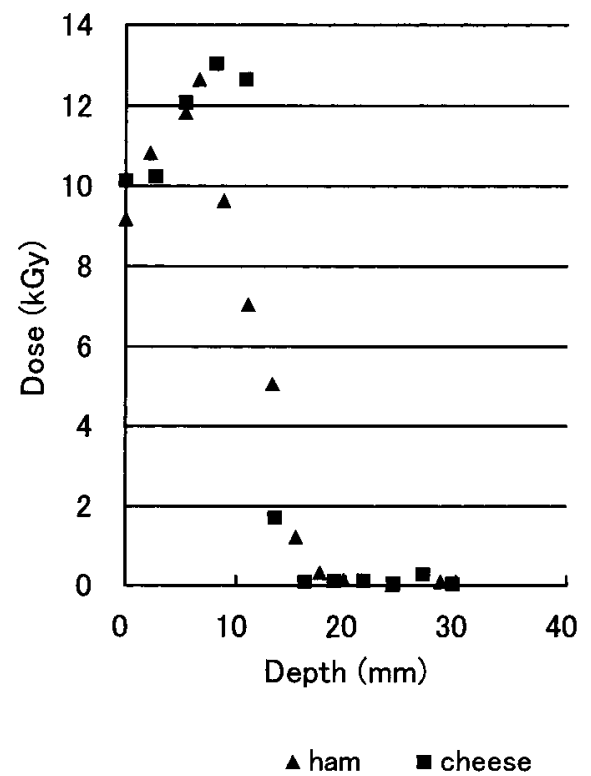

Fig. 6. Dose-Depth Profile of Ham and Cheese Samples

the acceleration power of EB. The maximum dose and shape of the distribution curve are affected by characteristics of the target, thickness of the target, and energy of the accelerator. While a dose-depth profile can be calculated under numerous conditions, the result will not corresponds to those for real materials. In this study, dose-depth profiles of ham and cheese samples were examined by placing RC dosimeters between slices of the food. The results are shown in Fig. 6. The horizontal axis corresponds to the absorbed dose and the vertical axis to the depth to which dosimeter was set.

As shown in the figure, the depth of the maximum dose in the foods was $8 \mathrm{~mm}$ and i.e., which value was 1.3 times that of the surface dose. The dose declines sharply, nearly reaching zero at a depth of $20 \mathrm{~mm}$. The densities of the foods were about 1 . Using the methods reported by ASTM ${ }^{8}$ (American Society for Testing and Materials) methods, the ranges of $5 \mathrm{MeV} \mathrm{EB}$ in the ham and cheese samples were calculated as approximately $18 \mathrm{~mm}$. These results neglected the thickness of dosimeters.

We conclude that in the course of developing chemical detection methods for irradiated foods, it is important to consider the dose-depth profile.

\section{REFERENCES}

1) a) The Radiation Application Development Association, The committee for dosimetry of high dose irradiation with electron beam (1990) Radiation dosimetry of Electron beams for radiation processing, Tijinnsyakan, Tokyo, pp. 19-23; b) Takeshita, M. (2000) Study Group for Quality Assurance of Herbal Medicines, Report \#1, the Japanese Association for the Promotion of Industrial Application of Irradiation, Kyoto, p. 84

2) The Radiation Application Development Association, The committee for dosimetry of high dose irradiation with electron beam (1990) Radiation dosimetry of electron beams for radiation processing, Tijinnsyakan, Tokyo, pp. 66-78.

3) Takeda, A. and Furuta, M. (1992) Evaluation of induced radioactivity in $10 \mathrm{MeV}$-electron irradiated foods, the Final Report of Food Irradiation Research Committee for 1986-1991, The Japan Radioisotope Association, Tokyo.

4) Kato, K. (1994) Examination committee for problems of induced radioactivity in accelerator at irradiation facility. Induction of radioactive materials and safe handling of them, The final Report of the Food Irradiation Research Committee for 19861991, The Japan Radioisotope Association, Tokyo.

5) The Radiation Application Development Association, The committee for dosimetry of high dose irradiation with electron beam (1990) Radiation dosimetry of electron beams for radiation processing, Tijinnsyakan, Tokyo, pp. 38-48.

6) Morimoto, K. (1993) Report of oversea-research commission of radiation dosimetry for radiation processing, The Radiation Application Development Association, Tokyo, pp. 25-36.

7) JIS Z4571, the alanine dosimetry system, Japan Industrial Standard Committee 2001.

8) ASTM (2000) Standard practice for dosimetry in electron beam facility for radiation processing at energies between $300 \mathrm{keV}$ and $25 \mathrm{MeV}$, Annual Book of ASTM 12.02, E1649, p. 856, American Standard and Testing Materials, West Conshohocken, U.S.A. 